\title{
Primary Care Confusion-Public Knowledge of NP and PA Duties and Their Information Gathering Behaviors
}

\author{
Evan K. Perrault, Ph.D. and Grace M. Hildenbrand, M.A. \\ Purdue University: Brian Lamb School of Communication, West Lafayette, IN, USA.
}

J Gen Intern Med 33(11):1857-8

DOI: $10.1007 / \mathrm{s} 11606-018-4580-\mathrm{x}$

(c) Society of General Internal Medicine 2018

\section{INTRODUCTION}

As the number of medical students entering primary care as a specialty declines, this deficit is increasingly being filled by mid-level providers such as physician assistants and nurse practitioners (PAs and NPs). Despite these providers' abilities to provide a similar quality of care as physicians, ${ }^{1}$ confusion likely exists regarding the kind of care these providers are able to deliver in comparison to the more widely understood physician profession. These problems are likely further compounded by the numerous combinations of alphabetical abbreviations appearing next to providers' names. Prior analyses of over 2000 primary care providers' online biographies ${ }^{2}$ found 181 unique combinations of alphabetical abbreviations next to names (e.g., PA-C, FNP-BC).

Little is known about the public's knowledge regarding what kinds of duties these mid-level providers can perform in comparison to physicians, nor the locations from which they seek information about providers before selecting one to visit. The current study seeks to fill this important gap.

\section{METHODS}

Between November 2017 and January 2018, we surveyed members of the US public from all 50 states via two methods: a large organization with over 12,000 employees allowed questions to be added to an annual employee survey, and Amazon Mechanical Turk where participants were paid $\$ .50$ for completing the survey.

Published online July 18, 2018
All participants answered questions regarding their knowledge of physicians', PAs', and NPs' abilities to prescribe medications, diagnose illnesses, and order lab tests. They also indicated where they seek information when having to choose a new healthcare provider, and the numbers of providers considered. This survey was approved by the Purdue University institutional review board.

A total of 4541 surveys were collected, and after removing surveys containing incomplete responses, 3948 completed surveys remained (response rate $87 \%$ ): employee survey $(n=2525)$, MTurk $(n=1423)$. Respondents included 2501 women $(63 \%)$ and 1266 men (32\%) with 181 not indicating (5\%); 3139 respondents were White $(80 \%), 200$ Black $(5 \%)$, 152 Asian (4\%), 125 Hispanic (3\%), and 332 indicating other or preferring not to respond (8\%). There were 597 respondents (15\%) aged 18-29 years; $1010(26 \%)$ 30-39 years; $781(20 \%)$ 40-49 years; 861 (22\%) 50-59 years; 533 (14\%) 60 years or older; and 166 (4\%) preferring not to specify.

\section{RESULTS}

Eighty-eight percent of respondents stated they seek information about healthcare providers before making a selection. Of these respondents, the most popular places they seek information are providers' online biographies, friends, and online patient comments (Table 1). The majority of respondents $(81 \%)$ indicated they consider between 2 and 5 providers before making a decision (Mean $=3.6$ providers).

Knowledge of the duties physicians could perform was extremely high. However, about half of respondents did not know PAs could prescribe medications and diagnose illnesses, and about one-third did not know NPs could prescribe medications and diagnose illnesses (Table 2). 
Table 1 Locations Participants Seek Information About Healthcare Providers: of Those Who Indicated They Seek Information $(n=$ 3488)

\begin{tabular}{ll}
\hline \hline Source & No. (\%) [95\% CI] \\
\hline $\begin{array}{l}\text { Online biographies provided by } \\
\text { healthcare providers }\end{array}$ & $2519(72)[71-74]$ \\
Friends & $2512(72)[71-74]$ \\
Patient comments online & $2268(65)[63-67]$ \\
Family members & $2100(60)[59-62]$ \\
Acquaintances/colleagues & $1983(57)[55-59]$ \\
Another healthcare provider & $1639(47)[45-49]$ \\
Other* & $241(7)[6-8]$ \\
\hline
\end{tabular}

*Common responses listed as "other" were insurance company, specific websites (e.g., Yelp, Healthgrades, Castlight), Google, and sites regarding lawsuits/malpractice claims

\section{DISCUSSION}

The current study's findings are concerning given that PAs and NPs are assuming a much larger role in primary care. ${ }^{3}$ Previous research has found many patients prefer physicians as their

Table 2 Participants' Knowledge of Care Providers $(N=3948)$

\begin{tabular}{|c|c|c|c|c|}
\hline & & \multicolumn{3}{|l|}{ Provider } \\
\hline & & Physician & $\begin{array}{l}\text { Physician } \\
\text { assistant }\end{array}$ & $\begin{array}{l}\text { Nurse } \\
\text { practitioner }\end{array}$ \\
\hline \multirow{4}{*}{$\begin{array}{l}\text { Able to } \\
\text { prescribe } \\
\text { medications }\end{array}$} & & $\begin{array}{l}\text { No. }(\%) \\
{[95 \% \mathrm{CI}]}\end{array}$ & $\begin{array}{l}\text { No. }(\%) \\
{[95 \% \mathrm{CI}]}\end{array}$ & $\begin{array}{l}\text { No. }(\%) \\
{[95 \% \mathrm{CI}]}\end{array}$ \\
\hline & True & $\begin{array}{l}3862(98) \\
{[97-981}\end{array}$ & $\begin{array}{l}1982(50) \\
{[49-52]}\end{array}$ & $\begin{array}{l}2598(66) \\
{[64-671}\end{array}$ \\
\hline & False & $\begin{array}{l}39(1) \\
{[0-1]}\end{array}$ & $\begin{array}{l}1110(28) \\
{[27-30]}\end{array}$ & $\begin{array}{l}745(19) \\
{[18-20]}\end{array}$ \\
\hline & Unsure & $\begin{array}{l}47(1) \\
{[1-2]}\end{array}$ & $\begin{array}{l}856(22) \\
{[20-23]}\end{array}$ & $\begin{array}{l}605(15) \\
{[14-17]}\end{array}$ \\
\hline \multirow{3}{*}{$\begin{array}{l}\text { Able to } \\
\text { diagnose } \\
\text { illnesses }\end{array}$} & True & $\begin{array}{l}3833(97) \\
{[97-98]}\end{array}$ & $\begin{array}{l}2025(51) \\
{[50-53]}\end{array}$ & $\begin{array}{l}2554(65) \\
{[63-66]}\end{array}$ \\
\hline & False & $\begin{array}{l}46(1) \\
{[1-2]}\end{array}$ & $\begin{array}{l}1043(26) \\
{[25-28]}\end{array}$ & $\begin{array}{l}738(19) \\
{[18-20]}\end{array}$ \\
\hline & Unsure & $\begin{array}{l}69(2) \\
{[1-2]}\end{array}$ & $\begin{array}{l}880(22) \\
{[21-24]}\end{array}$ & $\begin{array}{l}656(17) \\
{[16-18]}\end{array}$ \\
\hline \multirow{3}{*}{$\begin{array}{l}\text { Able to } \\
\text { order } \\
\text { lab tests }\end{array}$} & True & $\begin{array}{l}3844(97) \\
{[97-98]}\end{array}$ & $\begin{array}{l}2620(66) \\
{[65-68]}\end{array}$ & $\begin{array}{l}2917(74) \\
{[73-75]}\end{array}$ \\
\hline & False & 37 (1) & 509 (13) & $366(9)$ \\
\hline & Unsure & $\begin{array}{l}{[1-2]} \\
67(2) \\
{[1-2]}\end{array}$ & $\begin{array}{l}{[12-14]} \\
819(21)\end{array}$ & $\begin{array}{l}{[8-10]} \\
665(17) \\
{[16-18]}\end{array}$ \\
\hline
\end{tabular}

primary care providers, ${ }^{4}$ are less likely to want to visit PAs and NPs to get treated for minor concerns, and are likely to delay or not seek treatment if unable to see a physician right away. ${ }^{5}$ Healthcare organizations could be doing a better job helping prospective patients understand the similarities and differences between PAs, NPs, and physicians. Improving provider biographies - a primary source of information for prospective patients - might be one venue for educating about the duties that NPs and PAs can perform, which may lead to a greater willingness to utilize their services.

\section{Contributors: None.}

Prior Presentations: None.

Corresponding Author: Evan K. Perrault, Ph.D.; Purdue University: Brian Lamb School of Communication, West Lafayette, IN, USA (e-mail: perrault@purdue.edu).

Funders Startup funds provided by the College of Liberal Arts at Purdue University.

\section{Compliance with Ethical Standards:}

Conflict of Interest: The authors declare they do not have a conflict of interest.

\section{REFERENCES}

1. Lenz ER, Muldinger MO, Kane RL, Hopkins SC, Lin SX. Primary care outcomes in patients treated by nurse practitioners or physicians: twoyear follow-up. Med Care Res Rev. 2004;61(3):332-351.

2. Perrault EK. Campus health centers' lack of information regarding providers: a content analysis of division-I campus health centers' provider websites. Health Commun. 2018:33(7):860-866.

3. Morgan PA, Abbott DH, McNeil RM, Risher DA. Characteristics of primary care office visits to nurse practitioners, physician assistants and physicians in United States veterans health administration facilities, 2005 to 2010: a retrospective cross-sectional analysis. Hum Resour Health. 2012;10(42):1-8.

4. Dill MJ, Pankow S, Erikson C, Shipman S. Survey shows consumers open to a greater role for physician assistants and nurse practitioners. Health Aff. 2013;32(6):1135-1142.

5. Perrault EK. Improving student knowledge and perceptions of primary care providers at campus health centers. 2018. J Am Coll Health. https:// doi.org/10.1080/07448481.2018.1434781 